\title{
Long term Project in ASDEX Upgrade: implementation of ferritic steel
}

\author{
as in vessel wall
} \author{
Team \\ ${ }^{1}$ Max-Planck-Institut für Plasmaphysik, EURATOM Association, D-85740 Garching, Germany \\ ${ }^{2}$ Forschungszentrum Jülich, EURATOM Association, Germany
}

I. Zammuto ${ }^{1}$, L. Giannone ${ }^{1}$, A. Houben ${ }^{2}$, A. Herrmann ${ }^{1}$, A. Kallenbach ${ }^{1}$ and the ASDEX Upgrade

\begin{abstract}
A long term project is started at the ASDEX Upgrade (AUG) tokamak aimed at the exploration of the compatibility of reduced activation ferritic/martensitic steel (RAFM) with fusion devices. The topic is oriented towards the preparation of future experiments such as ITER with its test blanket modules and DEMO with its first wall designed with RAFM. The goal of the project is to gather experience with ferromagnetic materials inside the vacuum vessel, dealing with magnetic perturbations, both in plasma and magnetic probes, and facing up the additional magnetic forces acting on the supporting structures. The project envisages a stepwise replacement of the traditional graphite tiles with ferritic steel. For the time being, the main AUG actor is the inner heat shield (IHS), but further development can be imagined in the future. Since 2013, two of the 15 tile rows of the IHS have been replaced with ferritic steel and since now the experimental campaign has not suffered any particular problem related to the perturbation field induced by the steel tiles, as predicted by the calculation.

In the present paper, the preliminary study accomplished for the first phase for the evaluation of the forces and magnetic perturbation is reported, together with the further calculations required for the extension of the steel wall.
\end{abstract}

Keywords: electromagnetic modeling, reduced activation ferritic/martensitic steel, Eurofer, P92.

\section{Introduction}

Along with the well-known problems related to Tritium retention, high thermal load and fatigue, erosion, melting and so on, to tangle up the design of future fusion commercial reactors there is the neutron activation issue. In the present experimental devices and partially for ITER, the problematic of neutron activation for the wall material is not a driving parameter, but the problem will come up in reactor like DEMO and it should be properly addressed in an early stage. Research and development is concentrated on a reduced activation ferritic/martensitic steel (RAFM) developed years ago in Japan (F82H) and Europe (Eurofer). Since then, the materials have been extensively studied in laboratory characterizing their properties with and w/o neutron radiation [1]-[5]. Almost 10 years ago, an extensive scientific program has been carried out in the mid-size tokamak JFT-2M in order to characterize the plasma behavior with the F82H steel [6][7].

Since then, the use of the RAFM as in-vessel material has not been investigated in other tokamaks. Till 2013, when in AUG a long term project has been started with the plan to replace graphite tiles of the inner heat shield (IHS) with ferromagnetic material. The goal of the project is gather knowledge on the plasma wall interaction and experiences with ferromagnetic materials inside the vacuum vessel, dealing with magnetic perturbations, both in plasma and magnetic probes, and facing up the additional magnetic forces acting on the supporting structures. Therefore the work activity has been split in 2 main steps:
1. Replacement of just two tile rows symmetrically placed with respect to the plasma center position, in order to reduce the asymmetric field effects in plasma;

2. Extension of the metal wall replacing the whole graphite tiles, which would probably trigger a re-design of the heat shield support to stand the magnetic forces.

In the end of the 2013 the ferromagnetic steel tiles of the IHS have been installed. The martensitic steel P92 was selected for these activities as an alternative to Eurofer to facilitate the procurement and to cut expenses by a factor 5 for raw material. To cope for the lack of data available for the P92, a magnetic characterization of the material has been carried out, confirming the suitability of the choice (see par. 2).

In preparation of the first phase, an electromagnetic ANSYS 3D finite element model of one sector of AUG has been developed to pursue the magnetic perturbations and forces objectives. A corresponding structural model of the inner heat shield supporting structure was modelled to address the problem from a mechanical point of view. In vessel setup, design, finite element models and its outcomes are discussed at par. 3-4.

Possible extension of the ferromagnetic tiles through the IHS is discussed in the paragraph 5 followed by some conclusions.

\section{Material choice and characterization}

The high temperature steel P92 is an alternative material to Eurofer: it is easy and cheap to procure. The difference in term of cost is measurable: 50 euro $/ \mathrm{kg}$ for the Eurofer against 7/10 euro/kg for the P92. Eurofer can be delivered just in big quantities and EFDA/F4E are the exclusive contractor for this material. So, the easiest 
way, at least for the moment, is to install tiles made of P92. In Table 1 a comparison of the chemical composition and specification is reported. The first two columns are relative to the chemical specifications for Eurofer 97 [4] and Eurofer 97-2 [5] respectively.

Table 1 Chemical composition in weight percent of the Eurofer and $\mathrm{P} 92$ procured for AUG.

\begin{tabular}{l|lll}
\hline & Eurofer 97 & Eurofer 97-2 & P92 (AUG) \\
\hline $\mathbf{C}$ & $0.09-0.12$ & $0.09-0.12$ & 0.135 \\
$\mathbf{C r}$ & $8.5-9.5$ & $8.5-9.5$ & 9 \\
$\mathbf{S i}$ & $<500 \mathrm{ppm}$ & $<0.05$ & 0.38 \\
$\mathbf{M n}$ & $0.2-0.6$ & $0.2-0.6$ & 0.48 \\
$\mathbf{S}$ & $<0.005$ & $<0.005$ & 0.0010 \\
$\mathbf{P}$ & $<0.005$ & $<0.005$ & 0.017 \\
$\mathbf{N i}$ & $<50 \mathrm{ppm}$ & $<0.01$ & 0.37 \\
$\mathbf{M o}$ & $<50 \mathrm{ppm}$ & $<0.005$ & 0.52 \\
$\mathbf{V}$ & $0.15-0.25$ & $0.15-0.25$ & 0.200 \\
$\mathbf{N b}$ & $<10 \mathrm{ppm}$ & $<0.005$ & 0.064 \\
$\mathbf{A l}$ & $100<\mathrm{ppm}$ & $<0.01$ & 0.017 \\
$\mathbf{B}$ & $<0.001$ & $<0.002$ & 0.003 \\
$\mathbf{N}$ & $0.015-0.045$ & $0.015-0.045$ & 0.049 \\
$\mathbf{C o}$ & $<50 \mathrm{ppm}$ & $<0.01$ & \\
$\mathbf{C u}$ & $<50 \mathrm{ppm}$ & $<0.01$ & 0.07 \\
$\mathbf{Z r}$ & $<100 \mathrm{ppm}$ & $\mathrm{As}+\mathrm{Sn}+\mathrm{Sb}+\mathrm{Zr}<0.05$ & \\
$\mathbf{A s}$ & $<100 \mathrm{ppm}$ & $\mathrm{As}+\mathrm{Sn}+\mathrm{Sb}+\mathrm{Zr}<0.05$ & \\
$\mathbf{S b}$ & $<100 \mathrm{ppm}$ & $\mathrm{As}+\mathrm{Sn}+\mathrm{Sb}+\mathrm{Zr}<0.05$ & \\
$\mathbf{S n}$ & $<100 \mathrm{ppm}$ & $\mathrm{As}+\mathrm{Sn}+\mathrm{Sb}+\mathrm{Zr}<0.05$ & \\
$\mathbf{T a}$ & $0.06-0.09$ & $0.10-0.14$ & \\
$\mathbf{W}$ & $1.0-1.2$ & $1.0-1.2$ & \\
$\mathbf{T i}$ & $<0.01$ & $<0.02$ & \\
$\mathbf{O}$ & $<0.01$ & $<0.01$ & \\
$\mathbf{F e}$ & - & $b a l a n c e$ & \\
\hline & & & \\
\hline
\end{tabular}

In the last column the chemical analysis of the material purchased for the manufacturing of the AUG tiles is given. The specification for P92 and Eurofer at first sight looks quite similar.

To cope with the lack of data available in literature for the P92 magnetic behavior, some measurements were carried out. The vibrating sample magnetometer (Quantum Design) was used for both P92 and Eurofer samples. For this comparison the Eurofer 97-2 is used.

In Figure 1 the magnetization [Am $2 / \mathrm{kg}$ ] versus the temperature $[\mathrm{K}]$ curves for Eurofer and P92 are reported in pink and blue, respectively. The P92 has a slightly lower magnetization in comparison with the Eurofer. In Figure 2 the magnetic hysteresis curves for Eurofer and $\mathrm{P} 92$ are reported. The blue and red lines refer to the measured values of P92 and Eurofer, respectively. On the same chart the green line stands for the data available in literature [4].

\section{In vessel set up and IHS design}

The plasma center of AUG, in its position of equilibrium, has a vertical shift of in a range of $5-9 \mathrm{~cm}$.

Owing to reduce the asymmetric effect into the plasma, the two rows of tiles will be symmetrically placed with respect to the plasma position. Therefor a tile row will be replaced above the mid-plane and a row below it. In Figure 3 the tiles set up inside the vacuum vessel of AUG is illustrated: the replaced rows are highlighted in pink.

The supporting structure of the tiles consists of a welded structure made of a U-shaped channel with welded wings all along the poloidal extension and on both sides. Each tile is fastened to 2 adjacent wings by means of $2 \times$ M6 screws preloaded through a spring.

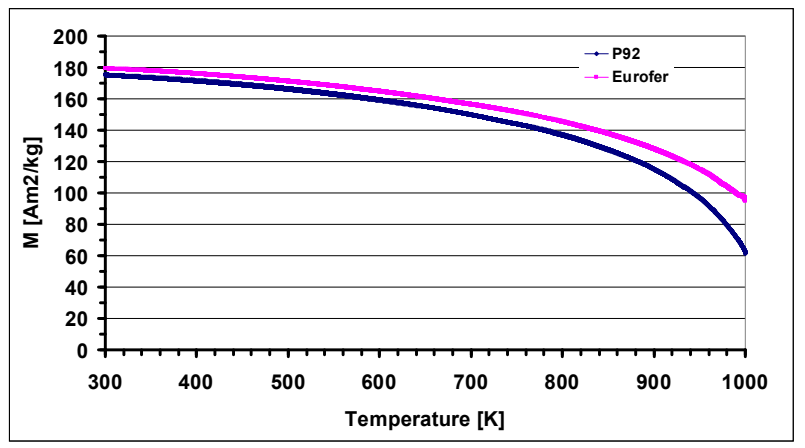

Figure 1 Comparison of the magnetization over the temperature: the blue line refers to the P92 and the pink line to the Eurofer.

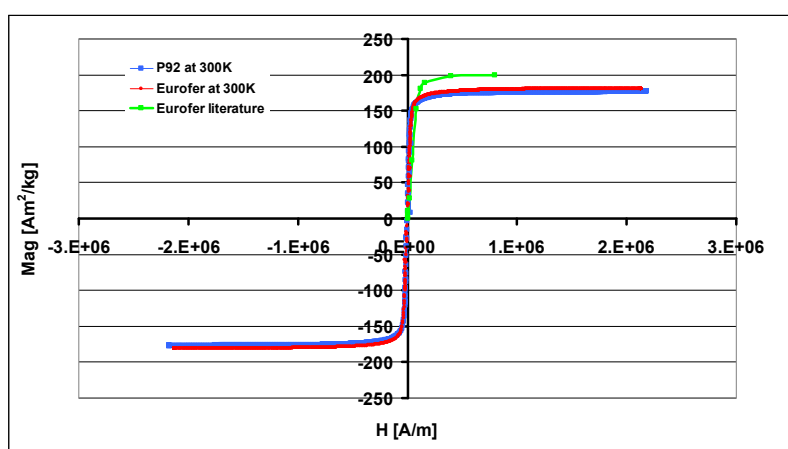

Figure 2 Hysteresis curve for the P92 and Eurofer at $300 \mathrm{~K}$ : blue line and pink lines refer to the measured value for the P92 and Eurofer samples. The green line is relative to the Eurofer values available in literature.

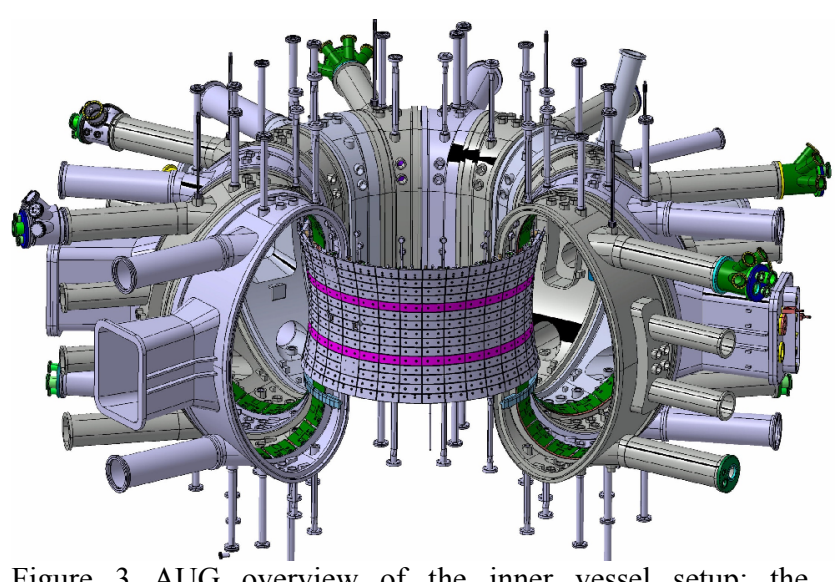

Figure 3 AUG overview of the inner vessel setup: the ferromagnetic tiles are highlighted in pink

The channel is connected to the vessel by means of stiffeners which are welded on the channel sides. The connection to the vessel is provided by means of $7 \mathrm{x}$ M12 screws. The channel is water cooled and the supporting structure is made of stainless steel. On the left side of Figure 5 the mechanical supporting structure of one sector of the IHS is depicted.

\section{Finite element model}

The magnetic scalar potential formulation is chosen for the FEM. Therefore the AUG coil system is entirely modelled while just one sector of IHS and vacuum are 
considered, applying on the sector edges the cyclic boundary conditions. In Figure 4 the FEM is illustrated. To ease the model view just one toroidal field coil is shown and the vacuum model is omitted. The plasma is modelled as a single toroidal ring where all the plasma current is applied; a better description of the plasma current distribution is negligible in term of magnetic perturbation.

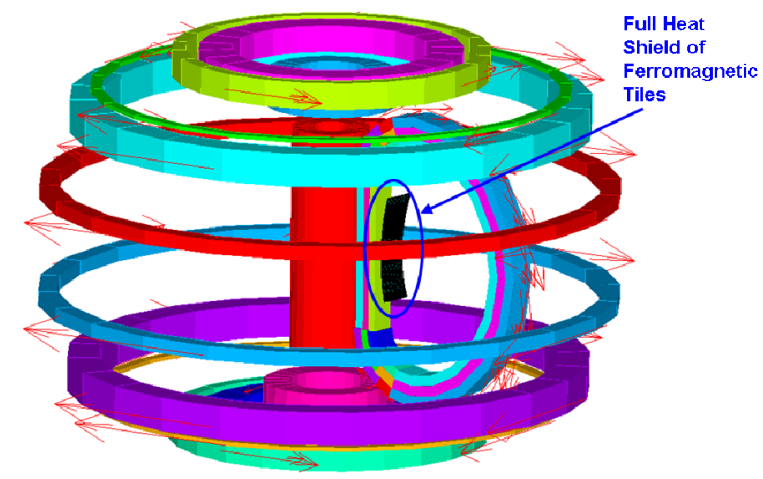

Figure 4 AUG FE mode: the poloidal field coils and just one toroidal field coil are depicted. The IHS made of P92 is in black. For sake of clarity the vacuum sector and the remaining toroidal field coils are omitted.

As first attempt the tiles have been modelled with a simplified shape, in order to reduce the computational time. It is assumed a constant thickness all over the toroidal tile extension. Fixing the poloidal extension of the tiles, the thickness was calculated so that the simplified tile has an equivalent volume of the real tile shape. In a second step, the tile FE model has been improve in order to better describe the electromagnetic forces acting on the structural components. In fact, in this case the forces transfer resulting from the electromagnetic analysis, to the structural mesh is accomplished in a node-to-node fashion, without requiring any further transformation of the obtained vector components.

This ensures highest accuracy of the force field for the structural calculation. The FE model has been run for a standard plasma configuration: shot \#28687 at $4 \mathrm{~s}$ is used $(\mathrm{Bt}=-2.5 \mathrm{~T}$, Iplasma $=1 \mathrm{MA})$.

\subsection{Results}

\subsubsection{Electromagnetic Forces and stresses in the structure}

The behavior of the tiles above and below the mid plane is quite similar. The predominant force is the radial one, which push the tile towards the center of the machine where the magnetic field is higher. A value of about 1.5 $\mathrm{kN}$ is calculated, while the vertical force is almost negligible, whit few dozens of $\mathrm{N}$. The latter is downward for the tiles in the upper mid-plane and it is upward for the other tiles.

The mechanical support has been checked against such forces and it withstands these forces without any problem.
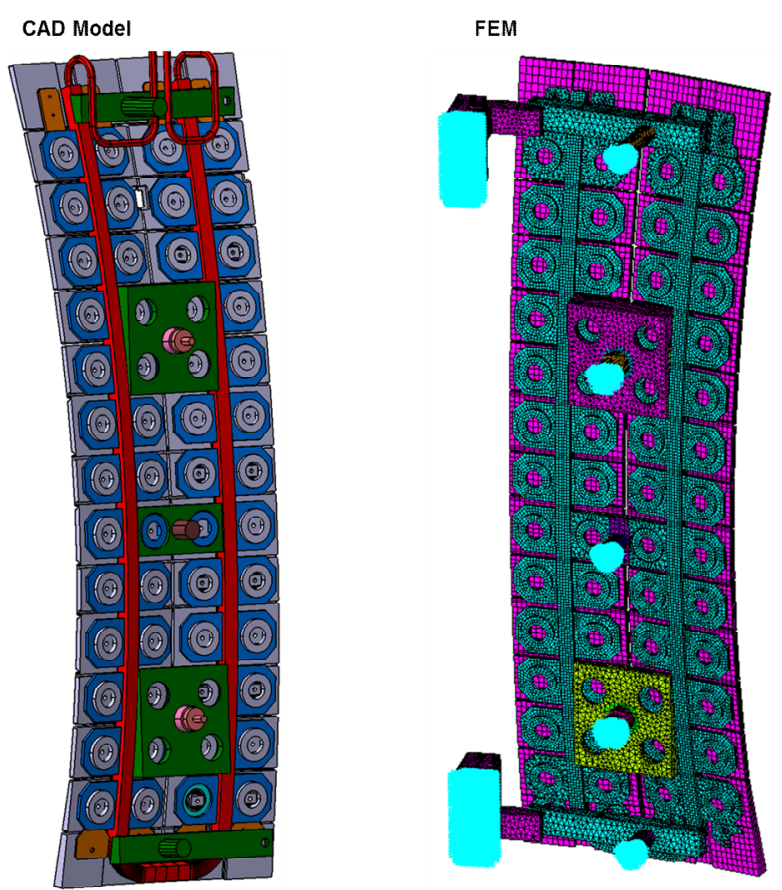

Figure $5 \mathrm{Cad}$ model on the left and FEM on the right for one AUG sector of the supporting structure of the tiles.

\subsubsection{Magnetic perturbations}

High magnetic field perturbations are expected in front of the ferromagnetic tiles and they vanish moving towards the plasma center: therefore poloidal magnetic fields with and without P92 are calculated along a radial path passing through the center of the tiles. The magnetic field perturbation is defined as the difference between the poloidal magnetic field without and with the magnetic field. In Figure 6 the magnetic perturbation in front of the tiles as function of the radial position is illustrated. A small difference is seen between the upper and lower row, solid and dash lines respectively. Dotted lines indicate the position of the plasma separatrix in its unperturbed configuration. The perturbation values in the plasma region is very small, it varies from 3 to $4 \mathrm{mT}$ for the upper and lower position respectively. Therefore no strong effects were expected on the plasma, as it was confirmed by the experimental campaign.

On the contrary to the plasma, the magnetic sensors are quite affected by the presence of such tiles. AUG is fully equipped with magnetic sensors distributed inside the poloidal extension of the vessel and some of them are sitting right behind the IHS. They are used either for feedback control or for plasma reconstruction. The greater number of the sensors is marginally affected by the tiles and the induced error is on the same order of the sensor precision. Just the closest sensors are suffering for the tile perturbation. Thanks to the saturation of the tiles induced by the toroidal field, the maximum value of perturbation is small with a maximum value of about 5 $\mathrm{mT}$ during plasma operation. 


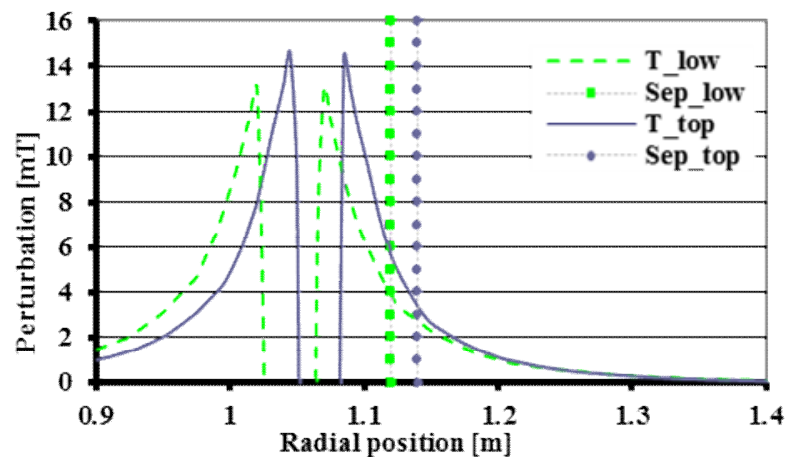

Figure 6 Poloidal magnetic field perturbation along radial path passing through the tiles: the dashed line and the solid line are referring to the tile below and above the mid-plane respectively. The dotted lines are defining the corresponding position of the separatrix for unperturbed plasma.

The sensors are more sensible to the tiles effect during the so called 'shot calibration', which is performed before each shot session. A daily control of the magnetic sensors together with the poloidal field coils is regularly done. During such shot the toroidal magnetic field coil system is off, therefore the nonlinearity effect of the ferromagnetic material is emphasized. Perturbations in such shot will vary from few $\mathrm{mT}$ up to tenth of $\mathrm{T}$. The real time magnetic equilibrium code handling the magnetic probes has been update as described in [8]. Fictitious currents are modelled in the code as function of the $\mu_{\text {eff }}$ calculated by the FEM. As reported in [8] a good agreement between the calculated magnetic field and the measured one reached with this approach.

\section{Feasibility of an extension of the ferromagnetic tile to the whole IHS}

Some preliminary study has been carried out, investigating the possibility to extend the ferromagnetic wall in AUG. The approach on evaluation of the magnetic perturbation both on plasma and magnetic probes has been already proved for the replacement of just 2 rows. Therefore the same logic will be extended also for this case.

For the mechanical issue, increasing the number of ferromagnetic tiles the forces exerted on the supporting structure will sensibly increase. The stiffness of the structure has been checked against such forces and as outcome Von Mises stresses in the connecting wings has been obtained. In those areas the stresses are up to 700 $\mathrm{MPa}$, well above the allowable stress. In Figure 7 Von Mises stress in the most stressed position is shown. If the whole IHS should be made of ferromagnetic material some technical details should be improved.

The mechanical issue on the IHS has to be addressed with attention and with a broader view deeming the various AUG upgrades in progress [9].

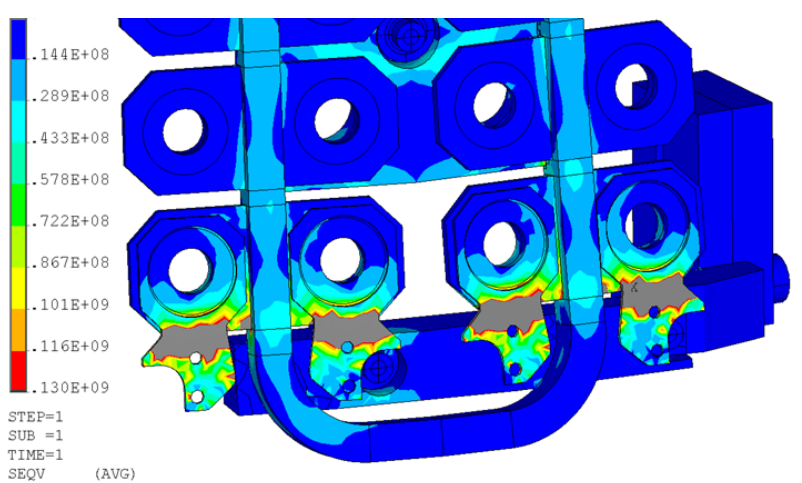

Figure 7 Von Mises [Pa] stress distribution in the supporting structure of the extreme tile of the IHS: in the gray areas the stresses are overcoming the allowable limit.

\section{Conclusion and outlook}

The experimental campaign started at the beginning of 2014 did not suffer any particular problem related to the ferromagnetic tiles. The plasma equilibrium reconstruction code and the code handling the magnetic probes were properly updated according to the precalculated $\mu_{\text {eff. }}$.

As next step a further graphite tiles will be replaced by $\mathrm{P} 92$ ones. To confirm that the P92 is a reasonable alternative to the Eurofer, some new tiles will be manufactured with Eurofer and installed during the next long shutdown. The material already in house was provided in kind by F4E. and new tiles will be manufactured in the next months.

\section{References}

[1] K. Mergia, N. Boukos - Structural, thermal, electrical and magnetic properties of Eurofer 97 steel, Journal of Nuclear Materials 373 (2008) 1-8.

[2] E. Materna-Morris et al, Structural material eurofer97-2, Characterization of ros and plate material: structural, tensile, Charpy and Creep properties, Final report on EFDA Task, March 2007.

[3] M. Marracci et al, Characterizaton of magnetic properties and losses of Fe-9Cr Steel Under Transient Conditions, IEEE Instrumentation and Measurements Technology Conference, Januar 2011.

[4] Tavassoli, Demo interim structural design criteria: A3.S18E Eurofer steel, draft

[5] L.V. Boccaccini et al, Technical note: selection and cost estimation for a material for a PFM in ASDEX Upgrade.

[6] T. Shibata et al, Advanced Material Tokamak EXperiment (AMTEX) on JFT-2M - Design, Fabrication, Installation and Conditioning of Inside Ferritic Steel Wall, IEEE 2002.

[7] K, Tsuzuki, High performance tokamak experiments with a ferritic steel wall on JFT-2M, Nuclear Fusion, IAEA 2003.

[8] L. Giannone et al, Enhancement of the real-time magnetic equilibrium on ASDEX Upgrade, EPS conference 2014.

[9] M. Schubert et al., Machine safety issue with respect of the extension of ECRH systems at ASDEX Upgrade, submitted to EC18/EPJ 